\title{
Solar Energy Conversion Toward 1 Terawatt
}

\author{
David Ginley (National Renewable Energy Laboratory, USA) \\ Martin A. Green (University of New South Wales, Australia) \\ Reuben Collins (Colorado School of Mines, USA)
}

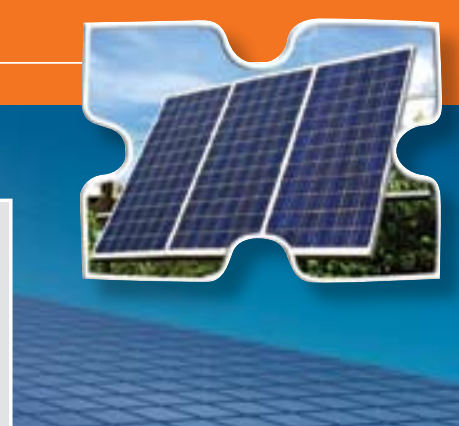

SEE ALSO SIDEBARS:

Concentrating Solar Power

Thermoelectrics

\section{Off-Grid Solar}

\section{GW Initiative} power production. The photovoltaics (PV) area is rapidly evolving based on new materials and deposition approaches. At present, PV is predominately based on crystalline and polycrystalline Si and is growing at $>40 \%$ per year with production rapidly approaching 3 gigawatts/year with PV installations supplying $<1 \%$ of energy used in the world. Increased cell efficiency and reduced manufacturing expenses are critical in achieving reasonable costs for PV and solarthermal. CdTe thin-film solar cells have reported a manufactured cost of $\$ 1.25 /$ watt. There is also the promise of increased efficiency by use of multijunction cells or hybrid devices organized at the nanoscale. This could lead to conversion efficiencies of greater than $50 \%$. Solar energy conversion increasingly represents one of the largest new businesses currently emerging in any sector of the economy.

\section{Introduction}

Harvesting energy directly from sunlight using photovoltaic (PV) technology or concentrating solar power (solar thermal energy conversion) is increasingly being recognized as an essential component of future global energy production. The decreased availability of fossil fuel sources and the realization of the detrimental long-term effects of emissions of $\mathrm{CO}_{2}$ and other greenhouse gases into the atmosphere are driving research and deployment for new environmentally friendly energy sources, especially renewable energy resources. An additional driving force is the increasing worldwide sensitivity toward energy security and price stability. Capturing even a small fraction of the 162,000 terawatts (TW) that reaches the earth would significantly impact the overall energy balance. PV systems, in addition, are portable and well-suited to distributed applications. The largescale manufacturing of photovoltaics is increasingly economically viable. The rapid expansion of manufacturing capability in PV components and the deployment of concentrating solar power (CSP) systems offers the potential for supplying a significant fraction (10\% without need for storage) of our energy demand with minimal environmental impact. (See accompanying sidebar by Mehos for additional detail.) In addition, it is clear that these technologies represent one of the next major high-technology economic drivers eventually succeeding microelectronics, telecommunications, and display industries. Truly achieving this goal will require materials-science-driven cost reductions, not just incremental cost reductions through economies of scale.

In 2004 , the average total worldwide power consumption was $15 \mathrm{TW}\left(1.5 \times 10^{13} \mathrm{~W}\right)$, with $86.5 \%$ from the burning of fossil fuels, according to U.S. Department of Energy statistics. In $2003,39.6$ quads $\left(1\right.$ quad $=1$ quadrillion $\mathrm{BTU}=1.055 \times 10^{9} \mathrm{GJ}$ 29.9 quad $=1 \mathrm{TW}$-year) of energy, largely from fossil fuels, was consumed to produce electricity just in the United States. After conversion losses, 13.1 quads of net electrical energy was output by power plants for general consumption. ${ }^{1}$ This amount of electricity could be produced by a $100 \mathrm{~km} \times 100 \mathrm{~km}$ area of high solar insolation, such as in the desert southwestern United States, covered with solar modules with a power conversion efficiency of $15 \%$. In order to meet the U.S. Department of Energy cost goal of $\$ 0.33 / \mathrm{W}$ or $\$ 0.05-0.06 / \mathrm{kWh}$ for utility-scale production, these modules would need to be manufactured at a cost of $\$ 50 /$ $\mathrm{m}^{2}$ or less. Goals for solar thermal power are comparable. Although the costs of modules are falling substantially, reaching these objectives with today's technology will require significant improvements in cell performance, as well as in the additional components making up the balance of solar systems. In addition, a variety of new technologies including thin films, thin silicon, organic photovoltaics, multijunction concentrator approaches, and next-generation nanostructured devices have the potential to significantly reduce the cost per watt.

With the recognition of the vast potential of photovoltaic technology, worldwide production levels for terrestrial solar cell modules have been growing rapidly over the past several years, with Japan recently taking the lead in total production volume (Figure 1). Current production is dominated by crystalline silicon modules (including both large-grain polycrystalline and single-crystalline materials), which represent $94 \%$ of the market. Devices based on silicon wafers, single- or polycrystalline, have been termed "first-generation" photovoltaic technology. These are fairly simple single-junction devices (diodes) that are limited by thermodynamic considerations to a maximum theoretical power conversion efficiency of $\sim 31 \%$ under direct AM1.5 sunlight. ${ }^{2}$ Solar cells and modules are usually characterized according to the IEC norm ${ }^{3}$ under standard test conditions (STC), which correspond to $1 \mathrm{~kW} / \mathrm{m}^{2}\left(100 \mathrm{~mW} / \mathrm{cm}^{2}\right)$ direct perpendicular irradiance under a global AM 1.5 spectrum at $25^{\circ} \mathrm{C}$ cell temperature. This means that an ideal silicon solar cell operating under direct sunlight converts approximately $30 \%$ of the illuminating solar radiation into electrical power, although actual cells suffer from parasitic losses. Current silicon solar cell design represents a considerable evolution beyond that of a simple single-junction device incorporating passivation of the surfaces, light trapping, and sophisticated anti-reflection coatings to help absorb most of 


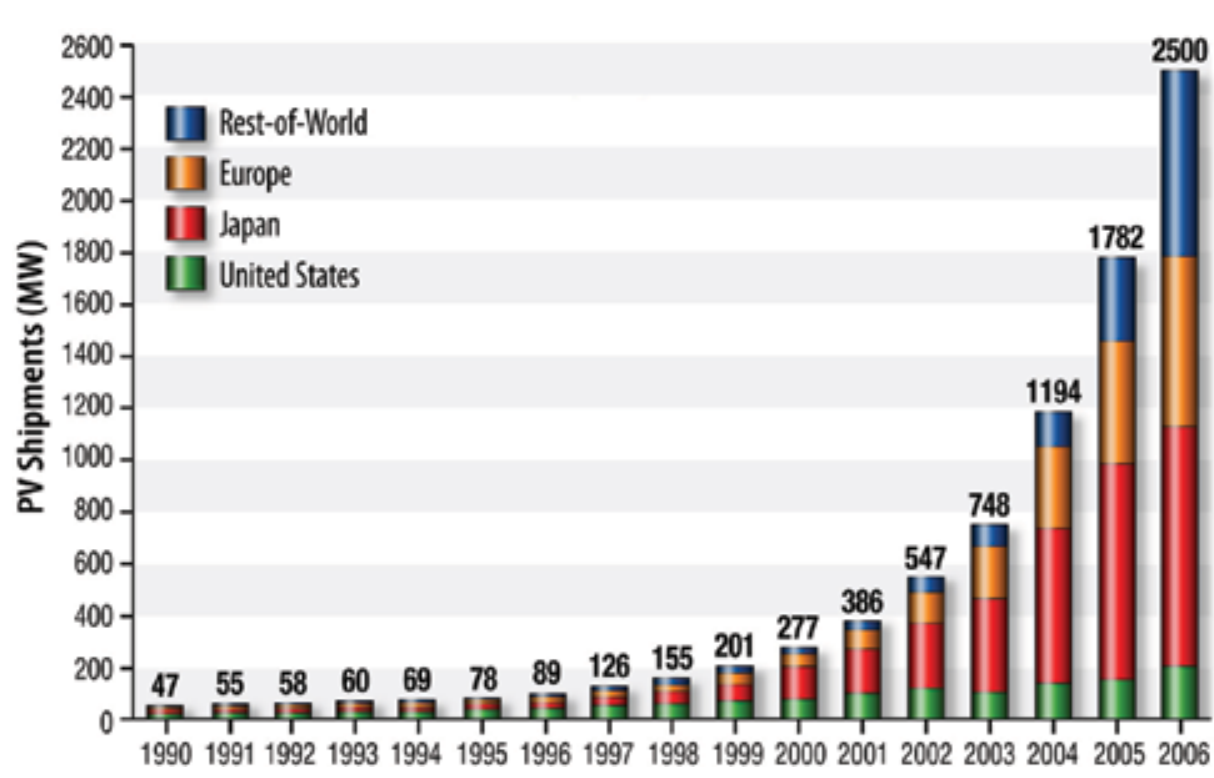

Figure 1. World photovoltaic module production (in megawatts), total consumer, and commercial per country (from PV News, Paul Maycock, Editor; February 2004). Most of this production is from crystalline or multicrystalline Si solar cells at present.

for devices based on $\mathrm{CdS} / \mathrm{CdTe}$ $\mathrm{Cu}(\mathrm{In}, \mathrm{Ga}) \mathrm{Se}_{2}$ (CIGS), and amorphous Si. These devices are fabricated using techniques such as sputtering, physical vapor deposition, and hot-wire chemical vapor deposition. Multijunction cells based on amorphous Si and amorphous SiGe alloys have been the most economically successful second-generation technology to date because of their ability to be fabricated at relatively low cost and to be integrated into electronics and roofing materials. These cells, together with other singlejunction amorphous $\mathrm{Si}$ devices, comprise $6 \%$ of the market not dominated by silicon.

However, it is not clear that second-generation technologies are capable of displacing silicon unless they can demonstrate significant cost reductions. The single counterexample has been the recent emergence of $\mathrm{CdTe}$ from First Solar, ${ }^{8}$ with a compelling low manufacturing cost in the range of $\$ 1.25 / \mathrm{W}_{\mathrm{p}}$. However,

the light in the wavelength range accessible to silicon and ensure that each absorbed photon leads to a carrier in the external circuit. Figure 2 illustrates a Sanyo high-efficiency Si heterojunction with intrinsic thin layer (HIT) solar cell that has an efficiency of up to $22.3 \%{ }^{4}$ In fact, for the bandgap of $\mathrm{Si}$, some current cells are pushing against the theoretical limit, so that it is methods to achieve significant cost reductions for the manufacturing of these sophisticated structures that are needed.

The progress in the efficiency of research-scale photovoltaic devices over the past several decades is shown in Figure 3. In nearly every technology, better understanding of materials and device properties has resulted in a continuous increase in efficiency. $\mathrm{Si}$, as already noted, is very close to its theoretical limit. In contrast, thin films such as amorphous $\mathrm{Si}(\mathrm{a}-\mathrm{Si}), \mathrm{Cu}(\mathrm{In}, \mathrm{Ga}) \mathrm{Se}_{2}$ (CIGS), and CdTe are all well below their potential maxima, and research efficiencies do not easily translate into production efficiencies. New technologies such as dye cells, organic photovoltaics, and third-generation concepts have just begun and have a long materials and development path ahead. These observations are apparent in the referenced compilation of highest confirmed cell and module efficiencies for many of the PV technologies. ${ }^{5}$

One key to the development of any photovoltaic technology is the cost reduction associated with economies of scale. This has been very evident in the case of crystalline silicon $(\mathrm{c}-\mathrm{Si})$ photovoltaics. Figure 4 shows the decrease in the cost of crystalline silicon photovoltaic modules as the production rate has increased, as well as predicted future costs for both wafer-based c-Si and the emerging technologies to be discussed. ${ }^{6}$ The current cost of $\sim \$ 4 / \mathrm{W}_{\mathrm{p}}\left(\mathrm{W}_{\mathrm{p}}=\right.$ watt peak $)$ is still too high to significantly influence energy production markets. Although it is difficult to determine exactly, best estimates are that costs for wafer-based Si panels will level off in the range of $\$ 1-1.50 / \mathrm{W}_{\mathrm{p}}$ in the next 10 years, ${ }^{7}$ substantially higher than the $\$ 0.33 / \mathrm{W}_{\mathrm{p}}$ target.

Thus, over the past decade, there has been considerable effort in advancing thin-film, "second-generation" technologies that do not require the use of silicon wafer substrates and can therefore be manufactured at significantly reduced cost. Steady progress has been made in laboratory efficiencies as can be seen in Figure 3 there are some residual concerns about the environmental effects of Cd, leading First Solar to adopt a "cradle-to-grave" approach, from sourcing of $\mathrm{Cd}$ from mining byproducts through recovery and recycling of $\mathrm{Cd}$ from used solar cells.

Although not based on thin films, concentrating solar applications using either photovoltaic cells (typically high-efficiency but costly groups III-V multijunction devices) or solar thermal collectors, take advantage of the relatively low cost of concentrating

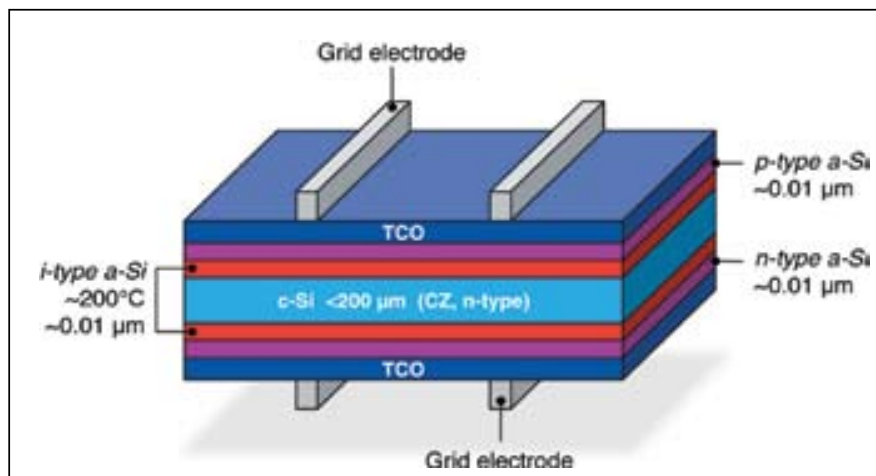

Figure 2. Illustration of the Sanyo heterojunction with intrinsic thin layer (HIT) cell that is based on crystalline $\mathrm{Si}$ and has demonstrated a $22 \%$ efficiency. TCO means transparent conducting oxide, typically used as a contact, and CZ indicates Czochralski-grown, which means pulled from the melt as a single crystal. The three types of amorphous silicon (a-Si) included in the cell differ in the types of dopants (or impurities) that have been added: $i$-type (or intrinsic) is undoped, $n$-type contains a dopant (such as phosphorous) that increases the number of free negative charge carriers (i.e., electrons), and $p$-type contains a dopant (such as boron) that increases the number of free positive charge carriers (i.e., holes). The types and amounts of impurities in the silicon affect its conductivity and other properties. 


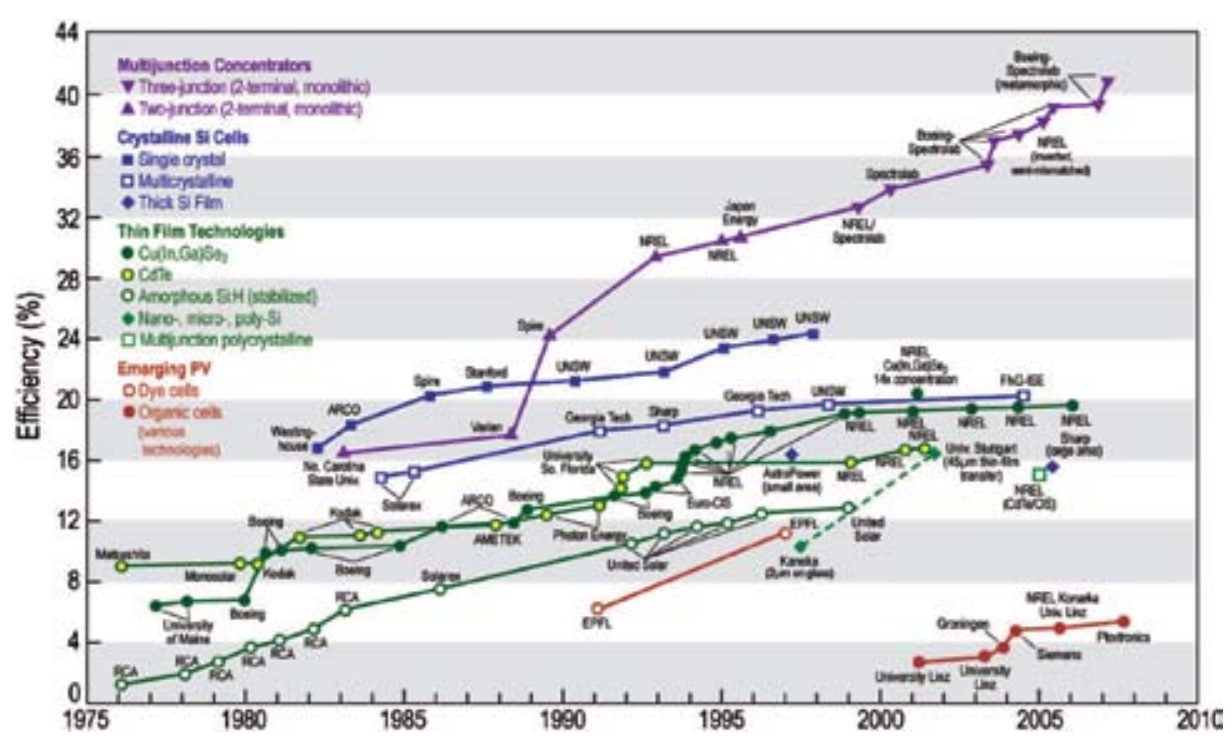

Figure 3. Progress of research-scale photovoltaic device efficiencies, under AM1.5 simulated solar illumination for a variety of technologies (as compiled by Larry Kazmerski, National Renewable Energy Laboratory).

processing approaches such as spray painting or inkjet printing.

The increasing manufacturing capacity in solar cells depicted in Figure 1, coupled with the uncertainties of fossil fuel energy sources, is clearly a significant driver for current photovoltaic technologies. Ultimately, however, there will be limits on how much the costs of current technologies can be reduced. Achieving truly competitive cost and efficiency might require breakthroughs even in mainstay technologies. This is represented by the red, black, and blue "learning curves" in Figure 4, where significant technological advances might be necessary to stay on the present trajectory at very high production volumes. This has driven more attention to the concept of third-generation PV technologies, as shown in Figure 5. ${ }^{9,10}$ Here, the primary focus is on those approaches to solar energy conversion that strive to achieve very high efficiencies by exploiting

optics to compensate for high-cost converters. These approaches are cost-competitive with most of the thin-film technologies.

In addition, an emerging set of devices employ organic- or dye-based absorbers/acceptors including the Grätzel cell, organic photovoltaic cells, and a number of third-generation concepts including intermediate-band devices (i.e., devices that use an impurity level in the bandgap of the semiconductor to essentially split the gap to absorb more solar radiation), quantum dot solar cells, and multiple exciton devices. Some of these devices (i.e., the Grätzel cell and polymer-based bulk heterojunction devices) have demonstrated initially attractive efficiencies of $>10 \%$ and $>5 \%$, respectively. Many of these devices can be fabricated with low-cost, solution-based, low-temperature, atmospheric-pressure

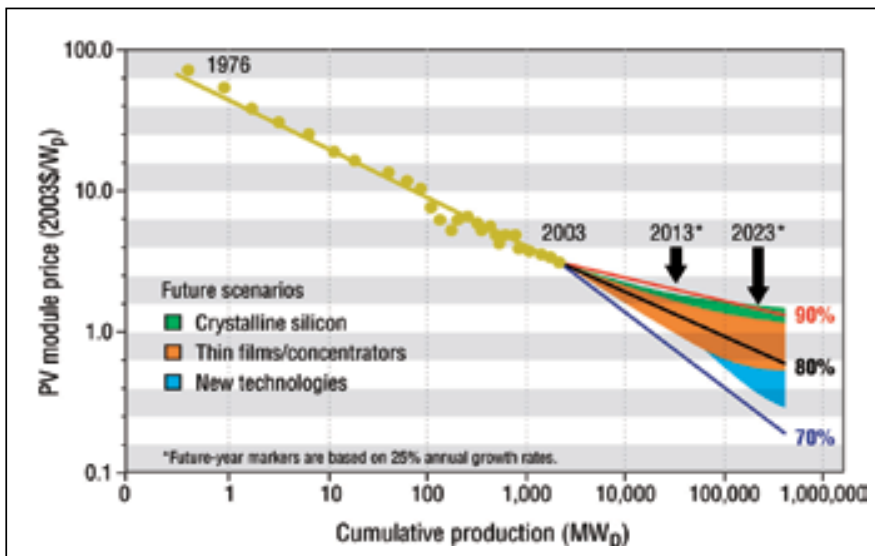

Figure 4. Historical and projected costs for wafer and film c-Si photovoltaic modules versus their cumulative production (in megawatts). Extrapolations for future technologies are also shown (from References 5 and 6). The $70 \%, 80 \%$, and $90 \%$ curves represent learning curves for the technology; the lower the percentage, the more rapid the learning, and the more rapid the price decrease with increasing production. processes that have smaller losses than a diode-based approach including hot-carrier collection, multiple electron-hole pair creation, and thermophotonics where the theoretical maximum efficiencies are in excess of the 31\% Shockley-Queisser limit for a single-junction device. In this case, the allowed cost of the cell can be higher. An alternative is to develop moderate-efficiency devices $(\sim 15 \%)$ at extremely reduced costs. This is where printable organic solar cell devices and other approaches that are not capital- or energy-intensive might become important. Achieving the goal of $<\$ 0.30$ per watt will require significant basic and applied science advancements over the next 20 years in a variety of technologies.

As we look to the future of solar energy, it is clear that materials science plays a critical role in this arena-in the near term for the improvement of Si, thin-film, and concentrator technologies and in the next 20-30 years for the development of thirdgeneration technologies. Although the focus here is on cell- and

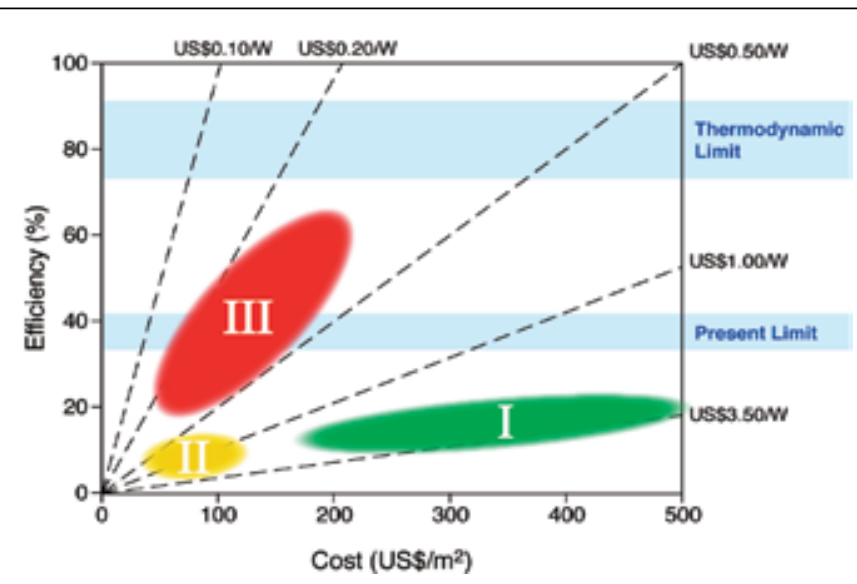

Figure 5. Cost-efficiency analysis for first- (I), second- (II), and third(III) generation PV technologies (from Reference 9). 
device-level materials science, it is important to keep in mind the broad range of materials considerations required for costeffective solar conversion systems. For example, solar systems must have anticipated installation lifetimes and warranties of up to 25 years. To accomplish this goal, all of the system components must be long-lived, low-maintenance, and stable. This requires solar cell packaging, contacting (bus structures), and support structures to be stable in a wide variety of climates with extremes in temperature, humidity, and wind, for example. Crystalline $\mathrm{Si}$ and stabilized amorphous $\mathrm{Si}$ have been able to meet these challenges. To date, the other thin-film technologies have not been commercially available long enough to evaluate their lifetimes, making the ability to perform accelerated aging on modules to predict stability a critical emerging area of solar science. Coupled closely to the development of improved costeffective photovoltaics is the eventual development of low-cost energy storage solutions, as discussed elsewhere in this issue (see the article and sidebar by Whittingham and the article by Crabtree and Dresselhaus). Also, as with all other technologies, PV modules contain valuable materials that will need to be recycled. Such considerations are generating interest in cradleto-grave or lifecycle approaches by some solar companies (such as First Solar mentioned earlier), whereby they install and will eventually remove and recycle their products. The natural resource needs, environmental issues of the various technologies, and impacts of recycling on the efficacy of technologies that use scarce or toxic materials are also important considerations as the solar energy sector moves forward.

\section{First-Generation Technologies}

First-generation technologies are primarily crystalline $\mathrm{Si}$ (including large-grain poly- and single-crystalline) materials. Figure 6 shows a 2 megawatt (MW) system installed at the Sacramento Municipal Utility District power station in Rancho Seco, Arizona.

The current technologies are rapidly evolving toward costs of $\$ 1-2 / \mathrm{W}_{\mathrm{p}}$ (see Figure 4). Key issues are Si feedstock supply, losses involved in preparing silicon wafers, and the development of lower cost high-throughput processing. To this end, a number of companies are exploring the use of ribbon-based technologies in which crystalline $\mathrm{Si}$ is grown as a thin sheet directly from molten $\mathrm{Si}$. This avoids the kerf loss (materials lost by sawing) associated with cutting blocks of polycrystalline

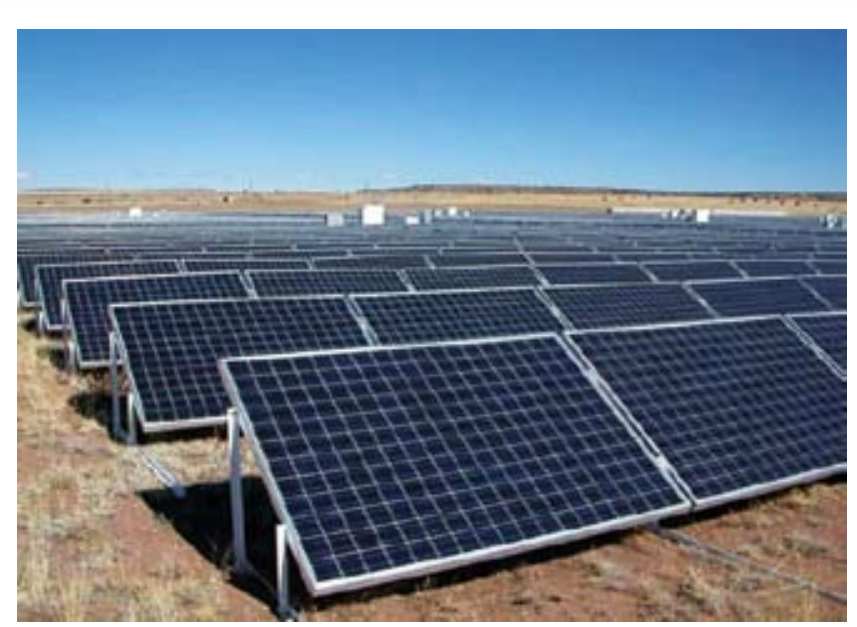

Figure 6. Two megawatt system installed at the Sacramento Municipal Utility District power station in Rancho Seco, Arizona. silicon or boules of single-crystal Si into wafers. To date, the materials produced by the ribbon approach have not yielded the efficiencies of wafer-based single-crystal materials, but their efficiencies are reasonable for commercial viability. Both the ribbon and boule technologies are striving to achieve thinner cells. A cell that is approximately 30 microns $(\mu \mathrm{m})$ thick and has an efficiency comparable to that of current cells (which are $100-200 \mu \mathrm{m}$ thick) would significantly reduce materials costs. Manufacturing such a cell would require new ways to process, contact, and handle such thin materials. For example, contacting could be done by inkjet noncontact printing instead of the screen-print approach currently employed.

\section{Second-Generation Technologies}

Second-generation technologies are those that have demonstrated practical conversion efficiencies and potentially lower costs per watt than crystalline silicon, but that have no significant market penetration at present. Commercialization of these approaches is typically in the early manufacturing phase. This group represents a fairly broad base of technologies, including thin-film photovoltaics, solar concentrators, solar thermal conversion, and the emerging field of organic photovoltaics that sits between second- and third-generation technologies. The potential for producing power at substantially reduced cost has recently been demonstrated by First Solar for CdTe solar cells, with a reported manufacturing cost of $\$ 1.25 / \mathrm{W}_{\mathrm{p}}$. Some of the key elements of thin-film inorganic PV approaches are summarized here, and further details can be found in a recent $M R S$ Bulletin issue. ${ }^{11}$

Amorphous silicon has been the most commercially successful thin-film PV technology to date, with $5-6 \%$ of the total PV market. Devices are typically single- or triple-junction designs laid down in multiple layers by vacuum deposition processes such as sputtering and plasma-enhanced chemical vapor deposition (Figure 7). The continued development of these multijunction cells is a materials challenge pushing the limits of materials synthesis and processing that is still not fully understood.

Amorphous silicon is attractive because its bandgap is quasidirect, leading to a larger absorption coefficient and hence thinner absorbing layers and less materials cost than crystalline silicon. That is, Si absorbs light less efficiently near its band edge (indirect gap) than does a-Si, in which absorption turns on very rapidly (direct gap). Alloying with Ge allows absorption to be tuned across a useful range of the solar spectrum as is shown in Figure 7. ${ }^{12}$ Amorphous Si, however, suffers from a lightinduced instability known as the Stabler-Wronski effect that causes the cell efficiency to degrade with time. Although the effect cannot, as yet, be eliminated, the extent of the degradation can typically be reduced to $10-20 \%$ of the as-manufactured (not aged) efficiency. Maintaining the initial efficiency of a-Si is one of the great materials science challenges facing this technology. Most of the work to limit this degradation is focused on controlling the hydrogen content and morphology of the film during growth. The same growth techniques used to deposit amorphous silicon can also be used to grow films consisting of nano- or microcrystalline silicon, either with or without an accompanying amorphous matrix, if the hydrogen content and deposition temperatures are changed, for example. These materials show crystalline, regularly ordered regions on a small length scale and usually form at increased process temperature. As grain size grows, the film begins to have more of the characteristics of polycrystalline silicon (also called polysilicon), with the energy gap becoming more indirect, thicker layers being required for complete absorption, and passivation becoming more important. Large-grain-size polycrystalline silicon thin films produced by solid phase crystallization of amorphous silicon are also being 
explored and are now in production (CSG Solar). In addition, combinations of polysilicon, amorphous silicon, and microcrystalline silicon are being explored to develop low-cost thin-film silicon solar cells on glass that could substantially reduce costs for large-area production.

\section{High-Efficiency Concentrators}

The highest efficiency solar cells known are multijunction cells based on GaAs and related groups III-V materials. Here, very sophisticated molecular beam epitaxy or metal-organic chemical vapor deposition (MOCVD) techniques are employed to grow a multilayer structure such as the triple-junction cell illustrated in Figure 8. These cells are too expensive for large-area applications but are usually used at the focus of mirrors or lenses that concentrate the solar light by a factor of 50-1,000. For maximum efficiency, the optic collection system must track the sun, which adds mechanical complexity to the system. However, by concentrating the solar energy by up to 500 times, the cost of the cell can be reduced to the point that the cost of the optics dominates. Thus, if inexpensive optics and trackers can be developed, the technology is very competitive with thin-film technologies. This is a significant materials challenge in its own right.

The efficiency of a singlejunction device is limited by transmission losses of photons with energies below the bandgap and thermal relaxation of carriers created by photons with energies above the bandgap. The purpose of a multijunction device is to capture a larger fraction of the solar spectrum while minimizing thermalization losses. By stacking cells in order of their bandgaps, with the cell with the largest bandgap at the top, light is automatically filtered as it passes through the stack, ensuring that it is absorbed in the cell most efficiently able to convert it. Another elegant simplification is that, if bandgaps are appropriately selected, all of the cells in the stack will generate close to the same current, so the cells can be simply interconnected in series. Figure 8 shows how a threejunction cell splits the solar spectrum, leading to a theoretical efficiency of $52 \%$ of the incoming energy converted to useful power for this particular combination of materials. The theoretical limit for multijunction devices where the number of junctions can be unlimited is above $60 \%$. The best efficiencies to date have actually been over $40 \%$, as recently reported for Spectrolabon multijunction cells with solar concentration. Achieving much higher efficiencies might require an increase in the number of junctions and/or integration of new absorber materials with more optimal bandgaps into the devices. ${ }^{13}$ The added complexity of concentrator systems, which must use tracking and concentrating optics, raises other issues such as appropriate site locations for tracking concentrators and the potential reliability/maintenance questions for active mechanical devices. The concentrator approach also collects little of the diffuse (scattered) solar radiation.

\section{Solar Thermal Conversion}

Historically, the most prevalent use of solar thermal systems has been flat-plate systems for domestic hot water or heating.
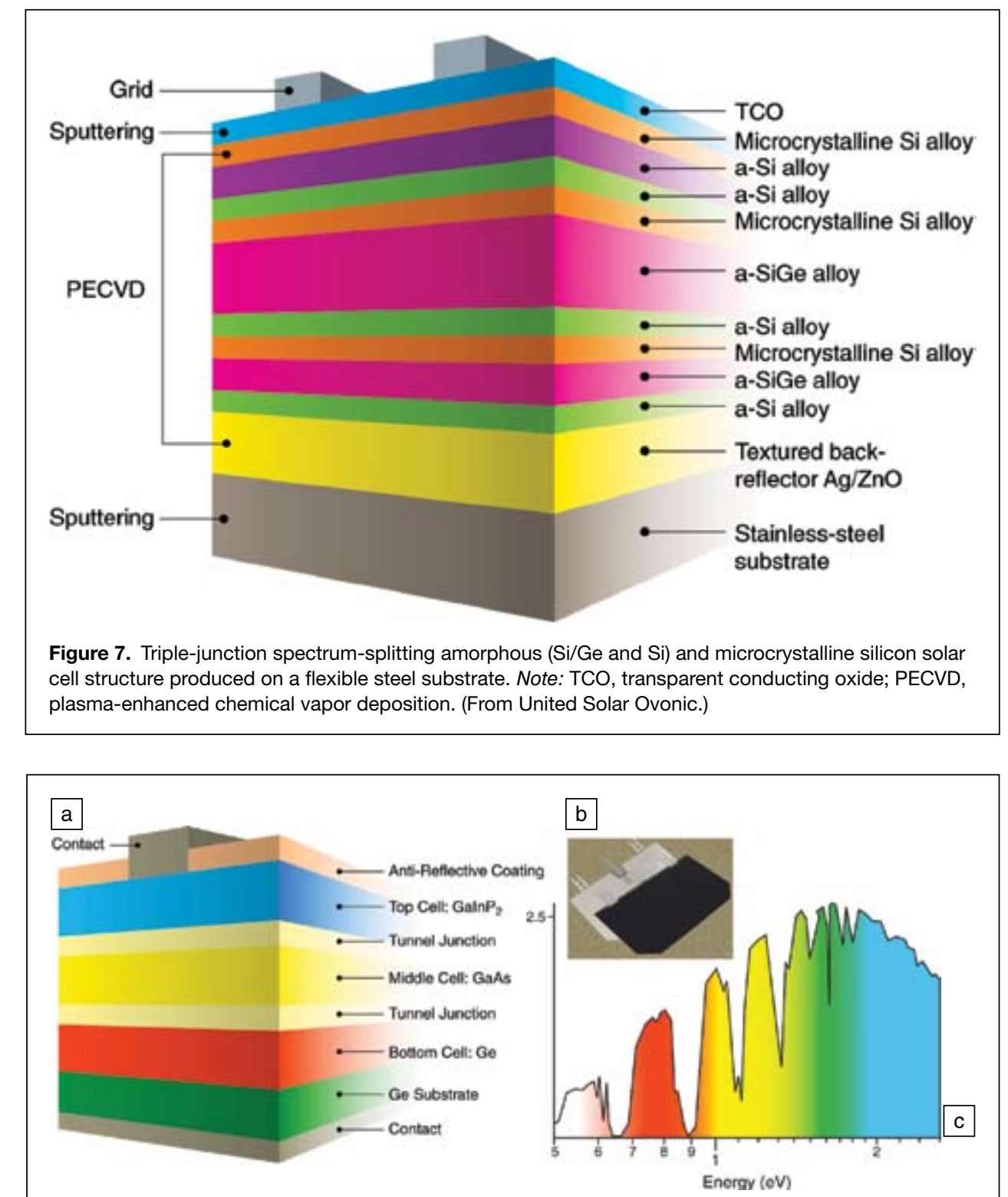

Figure 8. (a) Schematic of a possible configuration for a three- (triple-) junction device and (b) picture of a GalnP/GaAs/GalnAs cell with a demonstrated 31.3\% efficiency. (c) Solar spectrum illustrating the basic approach for spectrum splitting by a multijunction cell. A four-junction device with bandgaps of $1.8 \mathrm{eV}, 1.4 \mathrm{eV}, 1.0 \mathrm{eV}$, and $0.7 \mathrm{eV}$ would have a theoretical efficiency of $>52 \%$. 
Worldwide, this is perhaps the largest use of solar energy at present, estimated at up to 88 gigawatts $(\mathrm{GW})$ per year according to an Environment California report. In fact, solar hot water is required for new housing in some countries such as Israel. These systems can be either passive or active. A passive system typically uses no pumps and depends on a gravity feed, as shown in Figure 9. These systems represent an important energy-saving technology that can be applied almost anywhere.

More recently, large-area solar thermal systems have emerged as a promising option. Such systems use concentrating collectors for the solar radiation and heat a working fluid to a high temperature; the hot fluid can then be easily stored and utilized later to make steam to turn a turbine to generate electrical power. Figure 10 shows the solar power tower in California, which uses molten salts as a working fluid to produce $10 \mathrm{MW}$ of power.

Other approaches to central solar thermal power include parabolic dish systems and parabolic trough systems. All of the systems operate at relatively high temperatures, ideally $400^{\circ} \mathrm{C}$ or higher. This enables in situ storage and ready generation of steam. Key materials challenges lie in the development of working fluids for solar collectors that are stable throughout the broad temperature range experienced and have high heat capacities (amount of heat stored per molecule) but are still low in cost. New working fluids include polysilicones, nanostructured fluids, and molten salts. The other major area is the development of high-efficiency optical coatings for mirrors and for the

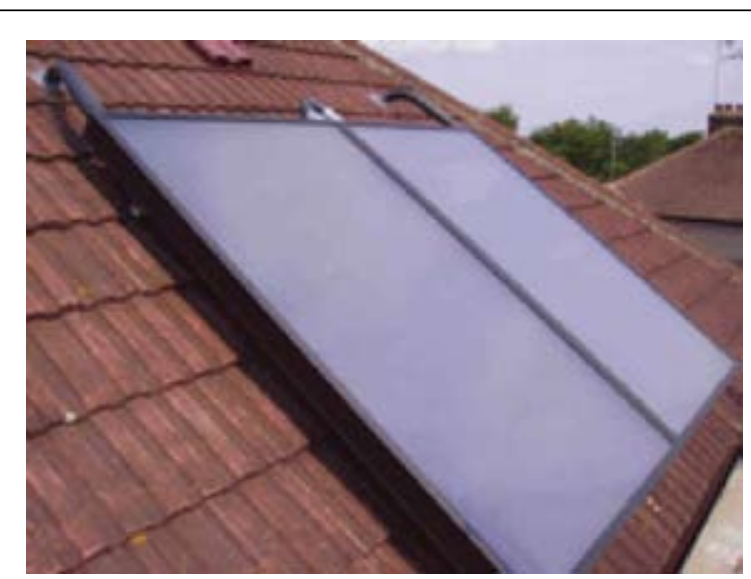

Figure 9. Passive solar hot water system on a residential roof top.

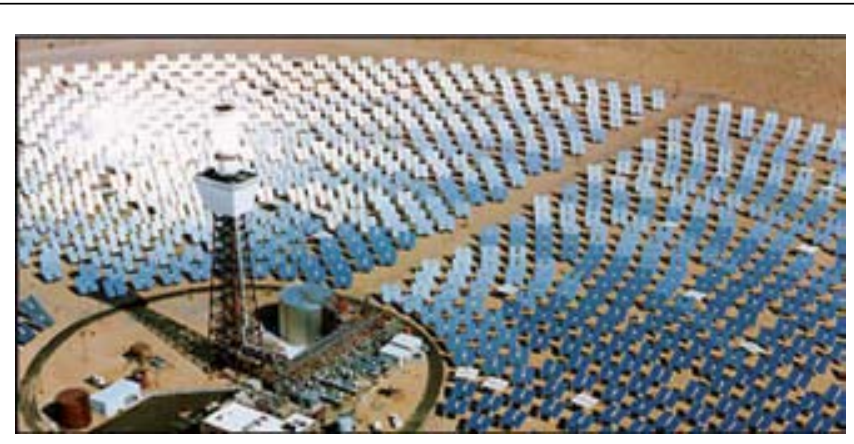

Figure 10. Solar 2 power tower in Barstow, California. The plant uses a combination of $60 \%$ sodium nitrate and $40 \%$ potassium nitrate as a working fluid and heat storage medium. absorption of solar energy. Estimates are that solar thermal electrical power production could be up to $7 \mathrm{GW}$ by 2015 . (See the sidebar by Tritt et al. for further information.)

\section{High-Efficiency Thin Films}

Figure 11 shows typical cross sections of polycrystalline $\mathrm{Cu}(\mathrm{InGa}) \mathrm{Se}_{2}$ (CIGS or CIS with no Ga) and CdTe solar cells. Laboratory efficiencies for the former are approaching $20 \%$ (Figure 3), ${ }^{14,15}$ and a large number of companies around the world are developing a variety of manufacturing approaches aimed at low-cost, high-yield, large-area devices that maintain laboratory-level efficiencies.

Materials challenges exist regarding each layer of these devices, as well as the interactions between layers, beginning with the search for improved transparent conducting oxide (TCO) contacts. Such contacts need to be made from low-cost, plentiful elements; have high conductivities and high transparencies in the visible spectrum; and allow for easy electrical isolation of devices.

Another example is provided by the thin CdS contact layer in both devices that also functions as a window for solar radiation. Because CdS absorbs in the blue wavelength range, it is important that this layer be thin. When the layer is too thin, however, pinholes between the TCO contact and the absorber layer create short circuits. This is especially problematic for CdTe cells, in which diffusion of sulfur into the CdTe layer during post-growth annealing further decreases the CdS layer thickness. The inclusion of thin buffer layers between the TCO and the CdS, such as a highly resistive transparent oxide, improves efficiency. ${ }^{15,16}$ The exact role of the buffer layer, whether it simply introduces resistance into short circuits or changes the interfacial energetics, is not well understood, and optimization of this interface is a critical need. As can be seen in Figure 11, although the absorbing CIGS and CdTe films are thin (ideally $1 \mu \mathrm{m}$ and $5 \mu \mathrm{m}$, respectively), the grain size for efficient devices is a large fraction of the thickness. In the case of CdTe, this large grain size is achieved by a post-deposition annealing in the presence of $\mathrm{CdCl}_{2}$ and oxygen, which promotes low-temperature grain growth. Finding controllable and manufacturable methods for performing this annealing treatment, or, better still, for incorporating it directly into the growth process, is an active area of research and development. The highest efficiency CIGS films are produced by physical vapor deposition, in which the grain microstructure is defined by a complex evolution from $\mathrm{Cu}$-rich to In- and $\mathrm{Ga}$-rich phases dur-

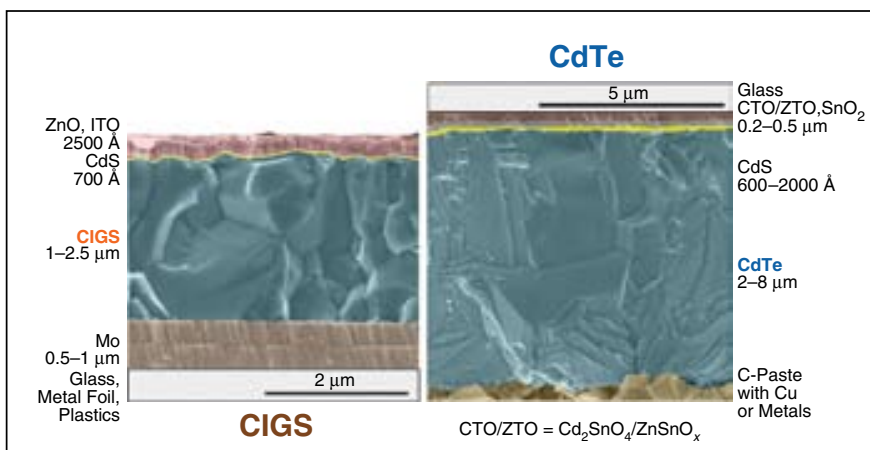

Figure 11. Cross-sectional scanning electron microscopy images of a $\mathrm{Cu}(\mathrm{InGa}) \mathrm{Se}_{2}$ (CIGS) solar cell (left) and a CdTe cell (right). The absorber layers are 1-2.5 and 2-8 $\mu \mathrm{m}$ thick, respectively. CTO (cadmium tin oxide) and ZTO (zinc tin oxide) are transparent conducting oxides used as transparent electrical contacts to the solar cell. 
ing growth. ${ }^{17}$ Reproducing this growth path with other more manufacturable deposition options is a major challenge. Incorporation of $\mathrm{Na}^{+}$ions, which occurs naturally as an aspect of growth on soda lime glass substrates, is also important to optimizing the CIGS device efficiency. Although there is no definitive explanation for the beneficial effects of $\mathrm{Na}, \mathrm{Na}$ is believed to reduce the resistivity of the film and possibly increase the grain size. ${ }^{18}$ The defect structures at the grain boundaries in both CIGS and CdTe are just beginning to be understood and are believed to play a dominant role in both minority carrier lifetime and carrier collection. Other absorber layer materials and processing issues that affect cell costs include the need to lower growth and processing temperatures which presently exceed $500^{\circ} \mathrm{C}$ for the highest efficiency films, and the desire to use thinner films without loss of absorption. The latter goal requires methods for increasing the optical path length through back-reflection, texturing, or light trapping.

For the back contact, significant challenges exist in understanding and improving the electronic and structural characteristics of these layers in both CIGS and CdTe devices. Delamination issues, for example, in CIGS cells are typically correlated with problems in the molybdenum back contact layer. Finally, we note that, although both CIGS and CdTe technologies have demonstrated good operating lifetimes, both are sensitive to moisture. They are presently protected by being sealed between glass plates. A thin-film encapsulation approach that provides a moisture barrier without compromising efficiency could be a significant advance. Beyond the simple thin-film cells depicted in Figure 11 is the potential to combine thin-film materials, $\mathrm{Si}, \mathrm{a}-\mathrm{Si}, \mathrm{CIS}$, and CdTe to form tandem cells that capture more of the solar spectrum, analogously to the previously mentioned multijunction concentrator structures. Such an approach would place even more stringent requirements on understanding and controlling the interfaces between dissimilar materials.

\section{Organic Photovoltaic Cells}

As detailed in a recent $M R S$ Bulletin issue, ${ }^{19}$ organic photovoltaics (OPV) represents a rapidly emerging device technology with the potential for low-cost non-vacuum-processed devices. OPV devices have rapidly moved from very low efficiency to efficiencies obtained by Plextronics and Konarka of 5.4\% and $5.2 \%$, respectively, as documented by the National Renewable Energy Laboratory (NREL). The key is that these devices combine polymers, small molecules, and inorganic nanostructures to build an excitonic-based structure. An excitonic solar cell first goes through an excited bound electron-hole state that subsequently generates charge carriers (which can do work in an external circuit) by decomposing at an interface. The charge carriers are then collected conventionally. Although the excitonic mechanism is different from the conventional PV mechanism, the theoretical efficiency is the same as for conventional semiconductor devices, with a cost structure similar to that for plastics processing, leading to the potential for significant reductions in cost per watt. The development of materials for OPV systems is being leveraged by the emergence of an organic electronics industry based on displays (organic light-emitting devices, OLEDs) and transistors..$^{20,21} \mathrm{~A}$ key aspect of OPV technology is that the organic small-molecule and polymer materials that are being investigated are inherently inexpensive; typically have very high optical absorption coefficients (very thin films work); are compatible with plastic substrates; and can be fabricated using high-throughput, low-temperature approaches by low-capital-cost roll-to-roll processes. $^{22}$ Thus, if efficiencies are comparable to or even slightly lower than those of existing technologies, there might be compelling cost arguments favoring OPV devices. Another important aspect of organic materials is their versatility. Organics exhibit a remarkable flexibility in the synthesis of basic molecules, allowing for alteration of a wide range of properties, including molecular weight, bandgap, molecular orbital energy levels, wetting properties, structural properties (such as rigidity, conjugation length, and molecule-to-molecule interactions), and doping. The ability to design and synthesize molecules and then integrate them into organic-organic and inorganic-organic composites provides a unique pathway in the design of materials for novel devices. Additionally, with the abilities to alter the color of the device, fabricate devices on flexible substrates, and potentially print them in any pattern, OPV cells can be integrated into existing building structures and into new commercial products in ways impossible for conventional technologies.

A number of key issues must be overcome for the ultimate success of OPV technology. One is the development of red absorbing molecules to utilize more of the solar spectrum without losing open-circuit potential. It is also necessary to develop optimized interfaces at the nanoscale that allow for exciton decomposition, charge transfer and optimization of the morphology of the organic constituent. Additionally, a key materials issue faced by the organic electronics community in general is the stability of the organic materials. It is encouraging that, for instance, automotive paints contain chromophores that are similar to molecules commonly used in OPV devices and that organic light-emitting displays are demonstrating acceptable lifetimes under high injection currents. Device degradation pathways stem largely from changes in morphology, loss of interfacial adhesion, and interdiffusion of components, as opposed to strictly chemical decomposition. Thus, careful design, prudent materials engineering, and improved encapsulation should substantially improve device lifetimes. In fact, the issue of encapsulation is important to all PV technologies and is becoming an increasingly active area of research. Development of encapsulants that are stable for 10-25 years with no yellowing and no diffusion of oxygen or water, that have low initial costs, and that are easy to process is a significant challenge. Increasingly, as for the OPV devices themselves, researchers are considering nanomaterial/polymer composites for sealants in which both elements scavenge impurites and slow diffusion. There are also efforts to develop new polymers with very little diffusion or photosensitivity to be the top and bottom layers for flexible PV devices.

\section{Third-Generation Technologies}

The term "third-generation photovoltaics" originally was coined to describe an "ultimate" thin-film solar cell technology. Features specified included high efficiency (derived from operating principles that avoided the constraints upon the performance of single-junction cells) and the use of abundant, nontoxic, and durable materials. In general usage, however, the term has been applied to any advanced photovoltaic technology ranging from organic cells to three-junction multijunction concentrator cells.

The multijunction approach discussed earlier is one of the best known and most investigated approaches meeting the aim of improving on the performance of single-junction cells. Efficiency ideally is limited merely by the number of cells in series; however, practical considerations tend to limit devices to between three and five junctions. Although the best cells do not meet the original definition of third-generation technology because they are not thin films and they use toxic and nonabundant materials, these disadvantages are not as severe in solar concentrating systems. ${ }^{23}$ Such concentrating systems closely resemble solar thermal electric systems, particularly dish-Stirling and power tower concepts, although the photovoltaic conversion unit is simpler, is potentially more reliable, and now has higher conversion efficiencies. (Note that a dish-Stirling system generates power by using parabolically arranged mirrors to reflect sunlight onto a 
small focal receiver, thereby heating a gas chamber connected to a piston and drive shaft. The drive shaft powers a generator that produces electricity to be distributed to a grid.)

The multijunction cell approach is also being extended to much less expensive and more exploratory materials systems. The approach potentially has the same efficiency advantages, for example, in organic and dye-sensitized solar cells, but in this case, the difficulty is finding a stable, low-bandgap cell that uses similar/compatible materials. Combinations of these materials with inorganic cells that have a lower absorption threshold have worked well, but such devices violate the basic guideline that the best cells need to be on top in a stacked cell design. Another multijunction approach explores the use of quantum confinement to increase silicon's bandgap and hence allow implementation of a higher temperature "all-silicon" tandem cell. ${ }^{24}$ By embedding $\mathrm{Si}$ quantum dots in a matrix of silicon oxide, nitride, or carbide, an increased optical bandgap has been demonstrated (Figure 12). Transport of photogenerated carriers is determined by dot density because, the closer the dots, the easier it is for carriers to tunnel between them. Stacks of either one or two of these quantum dot cells on top of a thin-film Si bottom cell have been proposed, with the effective bandgap of each cell determined by dot size.

Figure 13 shows the thermodynamic limiting efficiency for multijunction designs as well as a range of other options suggested as possible third-generation approaches. For diffuse sunlight, the thermodynamic limit on the terrestrial conversion of sunlight to electricity is about $74 \%$, although the limit for conversion approaches that are time-symmetric is somewhat lower at $68 \%$. A multijunction cell with a large number of cells in the stack can theoretically approach this limit, with limiting efficiency steadily dropping as the number of cells in the stack decreases, bottoming out at $31 \%$ for a single cell. (The best single laboratory cells made from Si or GaAs reach about $80 \%$ of this limiting efficiency.)

Hot-carrier cells also have close to the maximum theoretical performance potential. Rather than introducing complexity by stacking a large numbers of cells as in the multijunction approach, hot-carrier designs transfer the complexity to their operating physics (Figure 14). Although hot-carrier cells could be implemented as "simple" two-terminal devices and would almost certainly be very thin because of operating requirements, these requirements are severe. Unlike conventional cells, where photoexcited carriers quickly thermalize with the cell atomic lattice, a hot-carrier cell has to be designed so that the carriers are collected before this thermalization occurs. This suggests small transport distances and techniques for reducing the interaction between the carriers and the host lattice. Progress in nanostructural engineering aimed at controlling lattice vibrational properties might provide some opportunities here. Increasing the challenge further is the fact that careful attention has to be paid to the interface between the hot carriers and the outside world. Ideally, transfer should occur over only a narrow range of energies to prevent cooling of the hot carriers, again providing practical challenges. Resonant tunneling through quantum dots has been suggested as one way of meeting this requirement.

Next in efficiency in Figure 13 come the thermal approaches. A limiting efficiency of $54 \%$ applies to diffuse light conversion using such approaches. For direct sunlight conversion, such as for the concentrating solar power systems discussed earlier, this limiting efficiency jumps to $85 \%$ (but this value applies to only the fraction of the available light that is direct). The multiple processes in series in this case, as well as temperature constraints, limit practical efficiencies to only a fraction of this value.

Three other classes of approaches are also included in Figure 13. The first is based on multiple-step excitations between different energy levels deliberately introduced into a material. Again, nanostructures are being investigated as a means of introducing different ranges of available energies within a material so that twostep excitations are possible. The limiting efficiency is identical to that possible using optical upconverters, which could be placed at the rear of a cell. ${ }^{25}$ In this case, two low-energy photons, which are not able to cause excitations in the cell individually, can be combined in the upconverter in two-step excitation to produce one higher energy photon, which the cell can use. There is also some advantage in using down-conversion, ${ }^{26}$ where one high-energy photon produces two (or more) lower energy photons prior to the light entering the cell.

The final option shown in Figure 13 is impact ionization (see also Figure 15) but also includes multiple exciton generation (MEG), an approach that has created much recent interest. Evidence for the creation of up to eight excitons from a single photon has been found in $\mathrm{PbS}, \mathrm{PbSe}$, $\mathrm{PbTe}, \mathrm{CdSe}$, and InAs quantum dots, with more recent work 


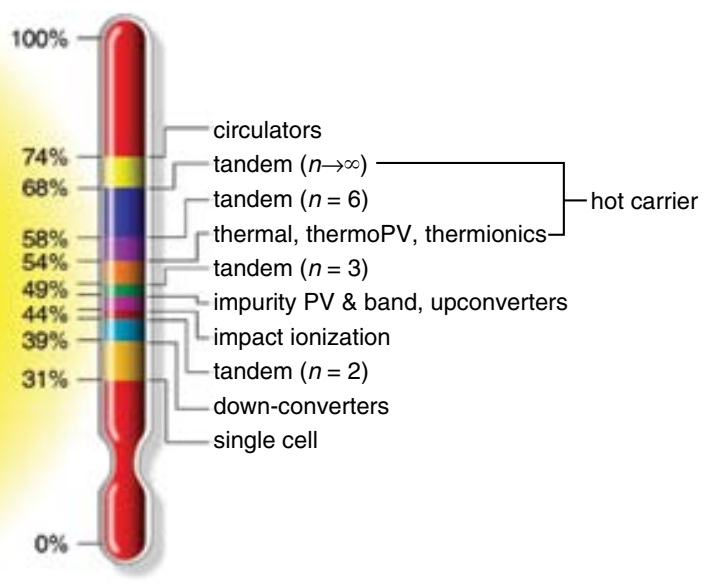

Figure 13. Third-generation options and thermodynamic limits on their efficiency. Upconverters include multi-excitonic approaches. Note: $n$ is the number of cells in the stack.

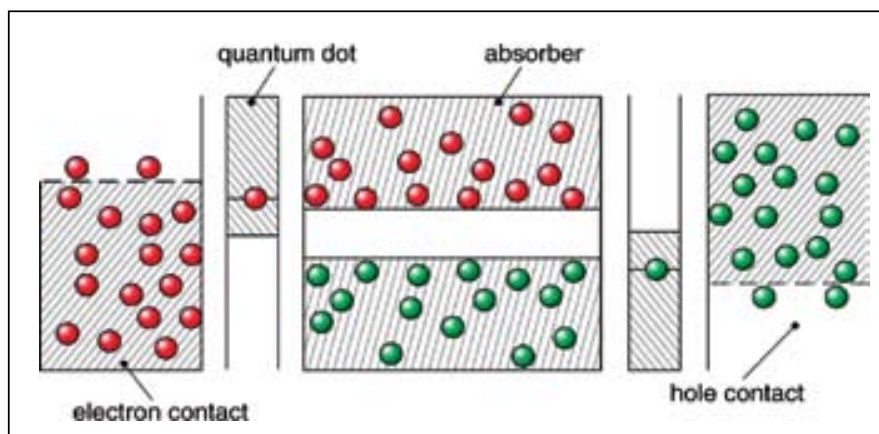

Figure 14. Hot-carrier cell schematic. Special properties are required for both the absorption volume and the carrier collection contacts.

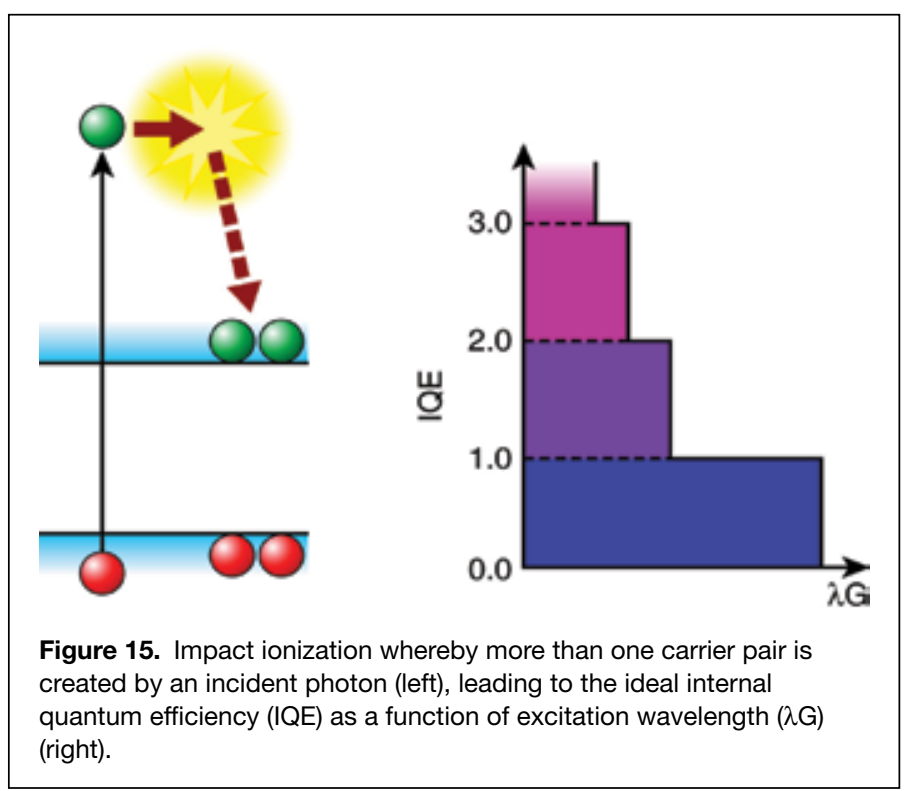

showing MEG in Si quantum dots as well. ${ }^{27}$ What is now needed is a way of converting these multiple excitons to electrons doing useful work.

It is clear that new materials, particularly nanomaterials, are closely linked to current third-generation research efforts. The flexibility offered by nanomaterials in the engineering of critical materials properties might allow for the eventual implementation of even the more challenging of these approaches.

\section{Summary}

As discussed herein, the solar power industry, both PV and thermal technologies, is on track to become an increasingly significant component of future global energy supplies. Although the industry is currently based on $\mathrm{Si}$, ultimately, Si might not be able to meet long-term cost goals, opening the door to thin films and solar thermal conversion. Significant materials challenges exist for these technologies as well, but they are nearing manufacturability on a large scale, as evidenced in the recent growth of CdTe production. New high-efficiency or low-cost technologies such as multijunction and organic-based devices are advancing rapidly and might have second- and third-generation embodiments. Finally, new very high-efficiency approaches to solar energy conversion offer the potential in the extended time frame to produce devices that can convert much larger portions of the solar spectrum. Given the anticipated market growth, nearly all of these approaches will have to be investigated in parallel to meet the demand.

Given the length of this article, the discussions of various solar technologies were of necessity brief, and adequate credit could not be given to all of the many leading scientists who have contributed to this field. For more information, interested readers are referred to the works previously cited as well as to the following resources on specific aspects of solar technology and the references cited therein: Si and thin-film inorganic photovoltaics, ${ }^{28-31}$ organic photovoltaics, ${ }^{32,33}$ third-generation photovoltaics, ${ }^{34,35}$ and solar thermal electricity. ${ }^{36}$

\section{References}

1. Energy Information Administration, Annual Energy Review 2003 (EIA, 2004; www.eia.doe.gov/emeu/aer/contents.html) (accessed January 2008).

2. W. Shockley, H.J. Queisser, J. Appl. Phys. 32 (3), 510 (1961).

3. International Electrotechnical Commission, "IEC Norm" (IEC-904-3, 1989).

4. S. Taira, Y. Yoshimine, T. Baba, M. Taguchi, H. Kanno, T. Kinoshita, H. Sakata, E. Maruyama, M. Tanaka, Proceedings of the 22nd European Photovoltaic Solar Energy Conference and Exhibition, Milan, Italy, 4, 932 (September 2007).

5. M.A. Green, K. Emery, D.L. King, Y. Hishikawa, W. Warta, Prog. Photovoltaics: Res. Appl. 15 (1), 35 (2007).

6. T. Surek, J. Cryst. Growth 275 (1-2), 292 (2005)

7. R.M. Margolis, Presented at NCPV Solar Program Review Meeting, Denver, CO, 2003.

8. D. Eaglesham, "First Solar, Inc. Announces 2006 Fourth Quarter and Yearend Financial Results" (First Solar, Phoenix, AZ, 2007; http://investor.firstsolar. com/releasedetail.cfm?ReleaselD=229824) (accessed January 2008).

9. M.A. Green, Physica E 14 (1-2), 65 (Amsterdam, The Netherlands, 2002).

10. M.A. Green, Photovoltaics for the 21st Century: Proceedings of the Electrochemical Society 2001-10, 3 (2001).

11. A. Slaoui, R.T. Collins, MRS Bull. 32 (3), 211 (2007).

12. B. Yan, G. Yue, J.M. Owens, J. Yang, S. Guha, Conference Record of the 2006 IEEE 4th World Conference on Photovoltaic Energy Conversion, Waikoloa, HI, 2, 1477 (2006).

13. F. Dimroth, S. Kurtz, MRS Bull. 32 (3), 230 (2007).

14. M.A. Contreras, M.J. Romero, R. Noufi, Thin Solid Films 511-512, 51 (2006). 15. X. Wu, Solar Energy 77 (6), 803 (2004).

16. M.A. Contreras, B. Egaas, K. Ramanathan, J. Hiltner, A. Swartzlander, F. Hasoon, R. Noufi, Prog. Photovoltaics: Res. Appl. 7 (4), 311 (1999).

17. J. AbuShama, R. Noufi, Y. Yan, K. Jones, B. Keyes, P. Dippo, M. Romero, M. Al-Jassim, J. Alleman, D.L. Williamson, $\mathrm{Cu}(\mathrm{In}, \mathrm{Ga}) \mathrm{Se}_{2}$ Thin-Film Evolution During Growth from (In, Ga) $)_{2} \mathrm{Se}_{3}$ Precursors (Mat. Res. Soc. Symp. Proc. Vol. 668, Warrendale, $P A, 2001)$.

18. A. Romeo, M. Terheggen, D. Abou-Ras, D.L. Batzner, F.J. Haug, M. Kalin, D. Rudmann, A.N. Tiwari, Prog. Photovoltaics: Res. Appl. 12 (2-3), 93 (2004). 
19. S.E. Shaheen, D.S. Ginley, G.E. Jabbour, MRS Bull. 30 (1), 10 (2005). 20. G. Collins, Sci. Am. 74 (2004).

21. D.M. de Leeuw, Phys. World 12 (3), 31 (1999).

22. S.E. Shaheen, R. Radspinner, N. Peyghambarian, G.E. Jabbour, Appl. Phys. Lett. 79 (18), 2996 (2001)

23. G. Hering, Photon Int. 10, 92 (2006).

24. M.A. Green, in Future Trends in Microelectronics: Up to Nano Creek, S. Luryi,

J. Xu, A. Zaslavsky, Eds. (Wiley Interscience, New York, 2007), p. 391

25. T. Trupke, M.A. Green, P. Wurfel, J. Appl. Phys. 92 (7), 4117 (2002).

26. T. Trupke, M.A. Green, P. Wurfel, J. Appl. Phys. 92 (3), 1668 (2002).

27. M.C. Beard, K.P. Knutsen, P. Yu, J.M. Luther, Q. Song, W.K. Metzger, R.J.

Ellingson, A.J. Nozik, Nano Lett. 7 (8), 2506 (2007).
28. R.W. Miles, G. Zoppi, I. Forbes, Mater. Today 10 (11), 20 (2007)

29. R. Messenger, D.Y. Goswami, H.M. Upadhyaya, T.M. Razykov, A.N. Tiwari, R. Winston, R. McConnell, in Energy Conversion, D. Yogi Goswami, F. Kreith, Eds. (CRC Press, Boca Raton, FL, 2007), p. 20.

30. M. Green, J. Mater. Sci. 18 (Suppl. 1), S15 (2007).

31. MRS Bull. 32 (3), (2007).

32. A.J. Mozer, N.S. Sariciftci, in Handbook of Conducting Polymers, T.A. Skotheim, John Reynolds, Eds. (CRC Press, Boca Raton, FL, ed. 3, 2007), vol. 2, p. 10.

33. MRS Bull. 30 (1), (2005)

34. G. Conibeer, Mater. Today 10 (11), 42 (2007).

35. A. Luque, A. Marti, A.J. Nozik, MRS Bull. 32 (3), 236 (2007)

36. D. Mills, Solar Energy 76 (1-3), 19 (2004).

\title{
Another Pathway to Large-Scale Power Generation: Concentrating Solar Power
}

\author{
Mark Mehos (National Renewable Energy Laboratory, USA)
}

\section{CSP's Great Potential}

Photovoltaics is not the only means of using sunlight to generate electricity. Another major solar technology is called "concentrating solar power" or CSP. CSP technologies use concentrating optics to generate high temperatures that are used to drive conventional steam or gas turbines. CSP is generally considered a central generation technology, rather than a source of distributed generation. That is, a large amount of power is generated in one location, with transmission and distribution to the various points of use, rather than generating small amounts of the power at numerous points of use. Because of this feature, CSP is predominantly a utility-scale source of power.

A 2005 study commissioned by the Western Governors' Association (WGA) looked at the solar resource and suitable available land in seven southwestern U.S. states (California, Arizona, Nevada, Utah, Colorado, New Mexico, and Texas) and calculated a capability of generating up to 6,800 gigawatts (GW) using CSP technologies - almost seven times the current electric generating capacity of the entire United States. It should be noted that this Geographic Information Systems (GIS) analysis determined optimal CSP sites with high economic potential by excluding regions in urban or sensitive areas (e.g., national parks), regions with low solar resource (e.g., those with insufficient hours of daily direct-normal radiation), and regions where terrain would inhibit the cost-effective deployment of large-scale plants (e.g., terrain that had more than a degree or two of slope). Other factors considered included land ownership, road access, local transmission infrastructure capabilities, and state policies and regulations. The WGA study found that, with a build out of only $2-4 \mathrm{GW}$ of CSP, the technology will be competitive with conventional natural-gas-fired combinedcycle plants with a cost of less than $\$ 0.10$ per kilowatt-hour. With increasing capacity and further research and development in thermal storage, CSP can be competitive with future coalbased generation, especially when considering the cost and performance impact of carbon constraints on future plants.

However, the southwestern United States is not the only area with great potential for CSP. Projects are under way in Spain and Northern Africa (e.g., Egypt, Algeria, and Morocco), with additional projects planned for Israel, the Middle East, Northern Mexico, and Australia. In total, over 40 utility-scale CSP plants are in construction or under various stages of development worldwide. These projects will lead to significant deployment in other regions with high solar resources, which includes areas with extended periods of sunny skies and relatively few clouds.

\section{Three Basic CSP Systems}

The three main types of concentrating solar power systems are parabolic trough systems, dish/engine systems, and power tower systems. Variants of these systems are also being considered, such as the compact linear Fresnel reflector system, which uses flat, rather than parabolic, mirrors with a Fresnel lens to concentrate the solar thermal energy.

Parabolic trough systems concentrate the sun's energy through long, rectangular, curved mirrors (see Figure 1). The mirrors are tilted toward the sun, focusing sunlight on a receiver,

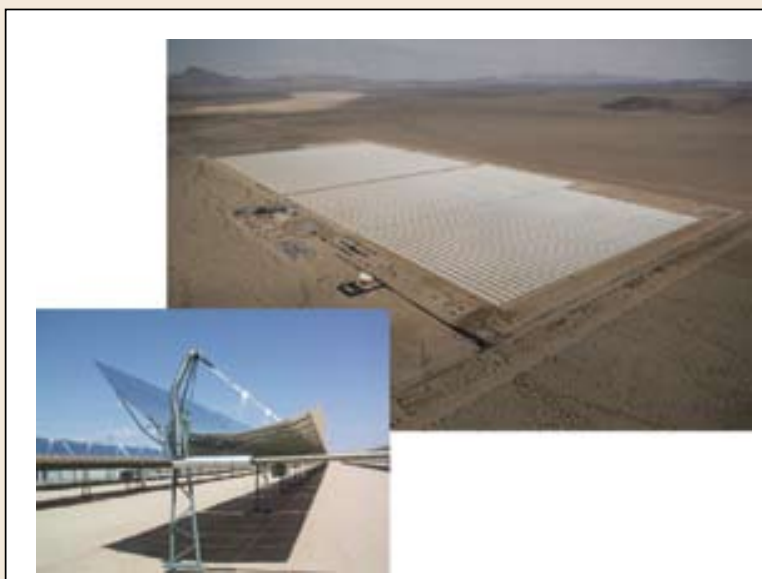

Figure 1. Aerial photograph of Acciona's Nevada Solar One, a $64 \mathrm{MW}$ parabolic trough power plant near Las Vegas, Nevada, that covers 280 acres (Credit: Acciona Solar Power). Inset: Closeup of an individual parabolic trough unit at Kramer Junction, California, showing the curved mirror and the receiver (Credit: Henry Price). 